\title{
Exercise Is Medicine! How to Safely Return to Sports after COVID-19? A Meta-Analysis and a Practical Flowchart for Cardiovascular Risk Assessment may Help you
}

\author{
Clea Colombo
}

Faculdade São Leopoldo Mandic Curso de Medicina, Campinas, SP - Brazil Editorial referring to the article: Cardiovascular risk assessment after COVID 019 infection before resuming sports activities-practical flowchart and meta-analysis

\begin{abstract}
"There are truly two different things: knowing and believing one knows. Science consists in knowing; in believing one knows lies ignorance."
\end{abstract}

Hippocrates

Exercise is medicine! Although this premise is well established, how to maintain this "treatment" for various diseases during the COVID-19 pandemic? This has been a concern of all doctors in the world since exercise contributes to the control of cardiovascular diseases and other comorbidities recognized as risk factors for a worse outcome in COVID-19. Moreover, it has already been shown that higher cardiorespiratory fitness is inversely associated with the likelihood of hospitalization due to COVID-19. ${ }^{1}$

In addition to the hygiene and social distancing measures necessary to prevent coronavirus infection, cardiovascular sequelae in individuals recovering from COVID-19 may contribute to the delay in resuming exercise. Cardiovascular complications, including myocarditis, are relatively common in patients affected by SARS-CoV-2. Although the occurrence of myocarditis as a consequence of COVID-19 was initially described in hospitalized patients with severe presentation of the disease, subsequent studies have reported its occurrence in individuals with mild COVID-19 and even in asymptomatic patients. ${ }^{2}$

\section{Keywords}

Exercise; Athletes; cardiovascular Diseases; Comorbidities; COVID-19; SARS-CoV-2, Sport; Sudden Death; Pandemic.
Thus, an alert was raised regarding the return to sports after COVID-19, since myocarditis is an important cause of sudden death in young individuals, especially in athletes. Since then, experts around the world have studied the occurrence of COVID-19-related myocarditis and discussed what would be the best way to return to exercise safely after recovering from the disease. ${ }^{3}$

The meta-analysis by Puga et al., ${ }^{4}$ makes a review of studies published until June 2020, to evaluate the prevalence of myocardial injury and propose a flowchart to assess cardiovascular risk in patients affected by COVID-19, to ultimately guide a safe return to exercise. Data from more than 6,000 hospitalized patients from China and the USA were divided according to the severity of the disease, based on requirement of intensive care unit (ICU) admission including non-survival. This interesting article, published in this issue, shows that there is a correlation between elevated troponin levels and the presence of myocardial injury. Also, the prevalence of myocarditis in hospitalized COVID-19 patients (21,7\%) increased with disease severity $-9.5 \%$ among patients who did not require intensive care unit (ICU) admission, and $57.7 \%$ among those who did not survive. These findings are similar to another recent review ${ }^{4}$ that reported a prevalence of myocarditis of $24.4 \%$ in hospitalized patients with COVID-19 and troponin elevation in $90 \%$ of them.

Puga et al., ${ }^{4}$ highlighted the lack of information in asymptomatic patients, including athletes, and reported a frequency of $1.9 \%$ of myocarditis in symptomatic non-hospitalized individuals (considered to have mild COVID-19), and many cases of severe COVID-19 in previously healthy individuals. Based on these, the authors discussed the urgent need for recommendations for resuming exercise after COVID-19 and proposed an evaluation based on the severity of the disease.

Mailing Address: Clea Colombo

R. Dr. José Rocha Junqueira, 13. Postal Code: 13045-755, Ponte Preta, Campinas, SP - Brazil.

E-mail: cleacolombo@gmail.com

DOI: https://doi.org/10.36660/ijcs.20210202 
The recommendations suggested by the Portuguese group are very interesting, and have similarities to the ones suggested by the Department of Ergometry, Exercise, Nuclear Cardiology, and Cardiovascular Rehabilitation (DERC) of the Brazilian Society of Cardiology (SBC) in the "Position Statement on Post-COVID-19 Cardiovascular Preparticipation Screening: Guidance for Returning to Physical Exercise and Sports - 2020" recently published. ${ }^{5}$ Both recommend a preparticipation cardiovascular screening of all individuals recovered from COVID-19, according to the clinical presentation of the disease (mild, moderate or severe) and the intensity of exercise practice.

Measurement of serum troponin Thas been considered important in the screening of patients with moderate and severe clinical conditions, as it can be a marker of myocardial injury. ${ }^{6}$ However, its use in athletes has been much discussed due to its variations during physical training and consequent lack of standard normal values. However, considering it is a low-cost and easy-to-perform test, I believe that determination of cardiac troponin $\mathrm{T}$ is useful in the evaluation of these individuals, if analyzed in both clinical and training context.

Resting 12-lead electrocardiography (ECG) is another low-cost method that can be very useful in cardiac evaluation of COVID-19 patients, as 90\% of patients with COVID-19 myocarditis have some electrocardiographic changes. ${ }^{6}$ However, like troponin, ECG must be carefully evaluated in athletes, because some findings may be confused with common physiological adaptations to training.

Exercise testing and transthoracic echocardiography $(\mathrm{ECHO})$ are also recommended in the preparticipation screening (PPS) of individuals with moderate or severe clinical presentation of COVID-19, as they reveal important information on functional capacity, exercise-related arrhythmias and changes in ventricular function and anatomy. The addition of cardiac magnetic resonance (CMR) is recommended for those who had a severe clinical presentation of the disease or who had abnormal results in other tests.

Cardiovascular assessment of athletes is always a challenge due to the peculiarities of the athlete's heart. The decision about including tests in the PPS is frequently controversial since misinterpretations can lead to unnecessary costs. This is not different in the PPS after COVID-19, as some of the changes considered as complications of the disease may also overlap with common findings of the athlete's heart. Therefore, the indications for tests, particularly CMR, have been debated.
Until the completion of the present review, data about myocarditis secondary to COVID-19 in athletes were lacking. Lately, studies including CMR in the evaluation of young athletes with COVID-19 have been published and reported a prevalence of diagnosed myocarditis ranging from $2.3 \%$ to $15 \%$ of athletes. This large variation is probably due to the difference in the sample studied, and mainly to the interpretation of the tests' findings. ${ }^{7-9}$ In CMR, myocardial injury or inflammation is detected by increased extracellular volume, T1 and T2 mapping, and presence of late gadolinium enhancement (LGE), which were the most used findings for the diagnosis of myocarditis in these studies. Although CMR is considered the gold standard for diagnosing myocarditis, these findings (2018 Lake Louise criteria) have been validated only in symptomatic individuals, and in these studies, a large proportion of the athletes were asymptomatic. Many of these findings, considered alone, do not have known long-term significance or prognosis, particularly in athletes.

Myocardial fibrosis, especially fibrosis of the right ventricular (RV) insertion point, has been described as a relatively common finding in some athletes, mainly endurance and master athletes. However, although some of the studies did not exclude this type of pattern from CMR findings and did not have a control group for comparison, most of them included predominantly young athletes and from different sports modalities. A large study involving 13 universities in the USA (The Big Ten COVID-19 Cardiac Registry), 8,9 evaluated 1,597 young athletes with a positive COVID-19 test who underwent CMR. The authors excluded fibrosis of the $\mathrm{RV}$ insertion point as an abnormality and reported the presence of myocarditis in about $2.3 \%$ of the athletes, with only $0.31 \%$ of them symptomatic. Changes in ECG, echocardiogram and troponin were also not significant among these cases, with an increase of 7.4 fold in the detection of myocarditis with the addition of CMR.

Thus, if on the one hand there is a recommendation of including CMR in the PPS for its capacity to detect myocarditis after COVID-19 and prevent sudden death in sports, on the other hand we must keep in mind that the interpretation of its findings may be controversial and should be done with caution. The physician's expertise with athletes and the performance of the tests in experienced centers are essential for a good result.

The main concern mentioned by the authors of the review is genuine and the theme is relevant. ${ }^{10}$ The Brazilian Society of Cardiology and Brazilian Society of Exercise and Sports Medicine Updated Guidelines for Sports and Exercise Cardiology - $2019^{10}$ 
and the European Society of Cardiology Position paper ${ }^{11}$ recommend PPS for sudden death prevention in sports. Based on this, after the pandemic, during which most people reduced or stopped exercising, the PPS should be performed before returning to sports practice. In the context of individuals recovering from COVID-19, medical assessment becomes essential, since there are reports not only of cardiac injury but also of different clinical sequelae. Moreover, we are still in the learning curve of the disease. PPS may be also useful in the readaptation to training, because the drop in performance is frequent and may be related from a simple deconditioning, peripheral dysfunction due to mitochondrial damage, to cardiac and pulmonary sequelae. A good evaluation minimizes the risks to the athlete's health, contributes to a gradual reintegration and adequate guidance for returning to the routine training.

The authors' idea of suggesting an assessment flowchart is very welcome, as it aims to help physicians in risk stratification and decision making. Experts play an important role to propose guidelines, using their knowledge and experience, aiming at the preservation of ethics and health. The authors also point out that the focus is on patient safety and that there is no data on the cost-effectiveness

\section{References}

1. Brawner CA, Ehrman JK, Bole S, Kerrigan DJ, Parikh SS, Lewis BK, et al. Inverse Relationship of Maximal Exercise Capacity to Hospitalization Secondary to Coronavirus Disease 2019. Mayo Clin Proc.2021 Jan;96(1):32-9. https://linkinghub.lsevier.com/retrieve/pii/ S0025619620311307

2. Puntmann VO, Gebker R, Duckett S, Mirelis J, Schnackenburg B, Graefe $\mathrm{M}$, et al. Left ventricular chamber dimensions and wall thickness by cardiovascular magnetic resonance: comparison with transthoracic echocardiography. Eur Heart J Cardiovasc Imaging. 2013 Mar 1;14(3):240-6. https://academic.oup.com/ehjcimaging/article-llookup/ doi/10.1093/ehjci/jes145

3. Bhatia RT, Marwaha S, Malhotra A, Iqbal Z, Hughes C, Börjesson M, et al. Exercise in the Severe Acute Respiratory Syndrome Coronavirus-2 (SARS-CoV-2) era: A Question and Answer session with the experts Endorsed by the section of Sports Cardiology \&amp; Exercise of the European Association of Preventive Cardiology (EAPC). Eur J Prev Cardiol. 2020 Aug 1;27(12):1242-51. https://academic.oup.com/eurjpc/ article/27/12/1242-1251/5950602

4. Puga L, Dinis P, Teixeira R, Ribeiro JM, Dores H, Gonçalves L. Cardiovascular risk assessment after COVID 019 infection before resuming sports activities-practical flowchart and meta-analysis. Int J Cardiovasc Sci. 2021.

5. Rathore SS, Rojas GA, Sondhi M, Pothuru S, Pydi R, Kancherla N, et al. Myocarditis associated with Covid-19 disease: A systematic review of published case reports and case series. Int J Clin Pract. 2021Jul 07; 021 Jul 7;(may):1-26. https://onlinelibrary.wiley.com/doi/10.1111/ijcp.14470

6. Colombo CSS de S, Leitão MB, Avanza AC, Borges SF, Silveira AD da, Braga F, et al. Posicionamento sobre Avaliação Pré-participação Cardiológica of the proposal. Less experienced physicians may follow the recommendations, but clinical decision should be individualized and consider local difficulties and regional differences. The use of additional tests in PPS was based on the clinical history of patients, and magnetic resonance imaging was recommended only for hospitalized patients and those with abnormalities detected in the initial PPS. The presence of symptoms in fully recovered individuals should always be considered as a red flag, but we must keep in mind that the occurrence of sequelae in asymptomatic patients is possible as well. Despite its variable and probably rare occurrence, post-COVID myocarditis does exist, and should not be underestimated by physicians.

In conclusion, PPS is recommended for a safe return to sports after COVID-19. However, complementary tests should be individualized, preferably performed in specialized centers and by physicians experienced with athletes, and their results carefully interpreted. Some clinical and test findings in the post-COVID-19 period must still be observed with caution and their significance evaluated in the long term, so that more robust guidelines can be defined. Only with much study and as time goes by, perhaps we can unite what we believe we know with what science will actually present to us.

após a Covid-19: orientações para retorno à prática de exercícios físicos e esportes - 2020. Arq Bras Cardiol. 2021 May 26; https://abccardiol.org/ article/posicionamento-sobre-avaliacao-pre-participacao-cardiologicaapos-a-covid-19-orientacoes-para-retorno-a-pratica-de-exercicios-fisicose-esportes-2020/

7. Daniels CJ, Rajpal S, Greenshields JT, Rosenthal GL, Chung EH, Terrin M, et al. Prevalence of Clinical and Subclinical Myocarditis in Competitive Athletes With Recent SARS-CoV-2 Infection. JAMA Cardiol. 2021 May 27;e212065 https://jamanetwork.com/journals/jamacardiology/fullarticle/2780548

8. Clark DE, Parikh A, Dendy JM, Diamond AB, George-Durrett K, Fish FA, et al. COVID-19 Myocardial Pathology Evaluation in Athletes With Cardiac Magnetic Resonance (COMPETE CMR). Circulation. 2021 Feb 9;143(6):609-12. https://www.ahajournals.org/doi/10.1161/ CIRCULATIONAHA.120.052573

9. Rajpal S, Tong MS, Borchers J, Zareba KM, Obarski TP, Simonetti OP, et al. Cardiovascular Magnetic Resonance Findings in Competitive Athletes Recovering From COVID-19 Infection. JAMA Cardiol . 2020 Sep 11;6(1):1168. https://jamanetwork.com/journals/jamacardiology/fullarticle/2770645

10. Ghorayeb N, Stein R, Daher DJ, Silveira AD, Ritt LEF, Santos DFP, et al. The Brazilian Society of Cardiology and Brazilian Society of Exercise and Sports Medicine Updated Guidelines for Sports and Exercise Cardiology - 2019. Arq Bras Cardiol. 2019;112(3):326-68.

11. Mont L, Pelliccia A, Sharma S, Biffi A, Borjesson M, Terradellas JB, et al. Preparticipation cardiovascular evaluation for athletic participants to prevent sudden death: Position paper from the EHRA and the EACPR, branches of the ESC. Endorsed by APHRS, HRS, and SOLAECE. Europace. 2016 Nov 4;19(1):euw243. https://academic.oup.com/europace/article-lookup/ doi/10.1093/europace/euw243 